\title{
Hereditary hyperferritinemia-cataract syndrome. Study of a new family in Spain
}

\author{
J. M. Ladero, A. Balas ${ }^{1}$, F. García-Sánchez ${ }^{1}$, J. L. Vicario ${ }^{1}$ and M. Díaz-Rubio \\ Department of Gastroenterology. Hospital Clínico San Carlos. Universidad Complutense. Madrid. ${ }^{~ C e n t r o ~ d e ~}$ \\ Transfusión. Servicio Madrileño de Salud. Consejería de Sanidad. CAM. Madrid, Spain
}

\begin{abstract}
The hyperferritinemia-cataract syndrome, inherited as a Mendelian dominant trait, is due to mutations in the 5' non-coding region of the ferritin light chain gene that modifies the shape of the IRE (iron responsive element) region, which loses its normal function of regulating the synthesis of ferritin light chains. Excess of light chains results in complexes that accumulate into the lens giving rise to early cataracts.

We present a Spanish family with seven affected members through three generations. A genetic study reveals a substitution of a single base $(\mathrm{C} \rightarrow \mathrm{T})$ at position 33 in the IRE sequence in the index case and in one affected brother, whereas a non-affected sister shows the normal sequence.

The hyperferritinemia-cataract syndrome was identified in 1995 and is still poorly understood. Clinicians should suspect it when treating any subject with early cataracts, even more if they are familial, or in patients with very high levels of ferritinemia without evidence of iron overload. There are no known consequences of the syndrome other than cataracts, and its proper diagnosis carries a favorable prognosis and eliminates the risk of unnecessary phlebotomies.
\end{abstract}

Key words: Hyperferritinemia. Cataract. Ferritin L chain. Iron responsive element.

Ladero JM, Balas A, García-Sánchez F, Vicario JL, Díaz-Rubio $M$. Hereditary hyperferritinemia-cataract syndrome. Study of a new family in Spain. Rev Esp Enferm Dig 2004; 96: 507-511.

Recibido: 15-01-03.

Aceptado: 17-02-04.

Correspondencia: José M. Ladero. C/ Modesto Lafuente, 46, 5 C. 28003 Madrid. e-mail: jladero.hcsc@ salud.madrid.org

\section{INTRODUCTION}

Variable hyperferritinemia levels are quite a common finding because ferritin is an acute phase reactant showing cytokine-inducible synthesis; these cytokines, in turn, are released in the course of inflammatory and tumor disorders (1). Extreme hyperferritinemia is a characteristic, although not pathognomonic or consistent, finding in hereditary hemochromatosis, usually in association with high plasma iron levels and transferrin saturation above 50 percent. Identification of mutations at the HFE gene, mainly homozygous C282Y or heterozygous C282Y/H63D, allows for the correct diagnosis of genetic hemochromatosis in more than $90 \%$ in Caucasians (2). Other genetic causes of iron overload have been recently reviewed (3), although with some discordances (4).

In 1995, Girelli et al., in Italy (5), and Bonneau et al., in France (6), identified a syndrome which associates hyperferritinemia with early-onset cataracts. The syndrome is inherited as a Mendelian dominant trait and is not associated with iron overload. To this date, several affected families have been identified in different European countries $(7,8)$, including Spain $(9,10)$, and in other nonEuropean regions (11), although the syndrome seems to be quite unusual. We report on a new affected family in Spain.

\section{CLINICAL REPORT}

The index case is a Spanish white woman born and living in Madrid (Spain). When she visited for the first time our outpatient clinic (May 2003), she was 44 years old. When she was 20, she was diagnosed with cataracts, and she was operated at 33 . When she was 42 year-old, hyperferritinemia was detected in a routine analysis and confirmed in a second control $(1130 \mu \mathrm{g} / \mathrm{mL})$. The remaining usual blood tests, including hemoglobin $(12.4 \mathrm{~g} / \mathrm{dL})$, plas- 
ma iron $(74 \mu \mathrm{g} / \mathrm{dL})$, transferrin saturation index (17\%), hepatitis $\mathrm{B}$ and $\mathrm{C}$ virus markers, autoantibodies, $\mathrm{Cu}$, ceruloplasmin and alpha-1-antitrypsin, were normal or negative. Abdominal ultrasonography was also normal. The patient was heterozygous and negative for H63D and C282Y mutations of the HFE gene, respectively. A consultant specialist prescribed 5 phlebotomies of $300 \mathrm{ml}$ each during the following 4 months: high ferritin levels persisted (1050 $\mathrm{ng} / \mathrm{mL})$ but serum iron $(46 \mu \mathrm{g} / \mathrm{dL})$, TSI $(12 \%)$ and hemoglobinemia $(10,9 \mathrm{~g} / \mathrm{dl})$ decreased significantly to infranormal levels. The patient was referred to our center.

Her mother and two of her three brothers had been diagnosed with early cataracts and subsequently with hyperferritinemia. The patient had two children; one of them was also diagnosed with cataracts and hyperferritinemia. The same occurred with one of the two children of one of the affected brothers. The other affected brother had an 8-year-old daughter who had not been studied yet. The remaining healthy sister had two nonaffected children (Fig. 1). No member of this family had been previously studied from a genetic point of view.

Hyperferritinemia was confirmed, normal values of serum iron and transferrin saturation were found, as well as hematological and biochemical parameters. A genetic study was performed in the index case, in one affected brother and in the non-affected sister. Gene amplification and sequencing approaches have been previously described for the IRE analysis of an unrelated Spanish family $(9,12)$. Both the index case and her affected brother were carriers of a single point mutation $(\mathrm{C} \rightarrow \mathrm{T})$ at position 33 of IRE region in the L-chain ferritin gene. The non-affected sister did not show any mutation in the studied DNA fragment.

\section{COMMENT}

Hereditary hyperferritinemia-cataract syndrome is a Mendelian dominant disorder due to a mutation in the 5 ' non-coding region of the ferritin L-chain gene, that maps at $19 \mathrm{q} 13.3-\mathrm{q} 13.4$ (13). This region, known as IRE (iron responsive element), interacts with two cytoplasmic proteins, known as iron regulatory proteins (IRPs). In iron deficiency status, IRPs acquire a high affinity for IRE, thus inhibiting the synthesis of L-ferritin chains. This interaction takes place on a loop structure formed by a 5 base-pair motif at an end of IRE (14).

Mutations in the loop or the adjacent stem structure prevent or decrease the interaction between IRE and IRPs, giving rise to a synthesis of ferritin L-chains that is

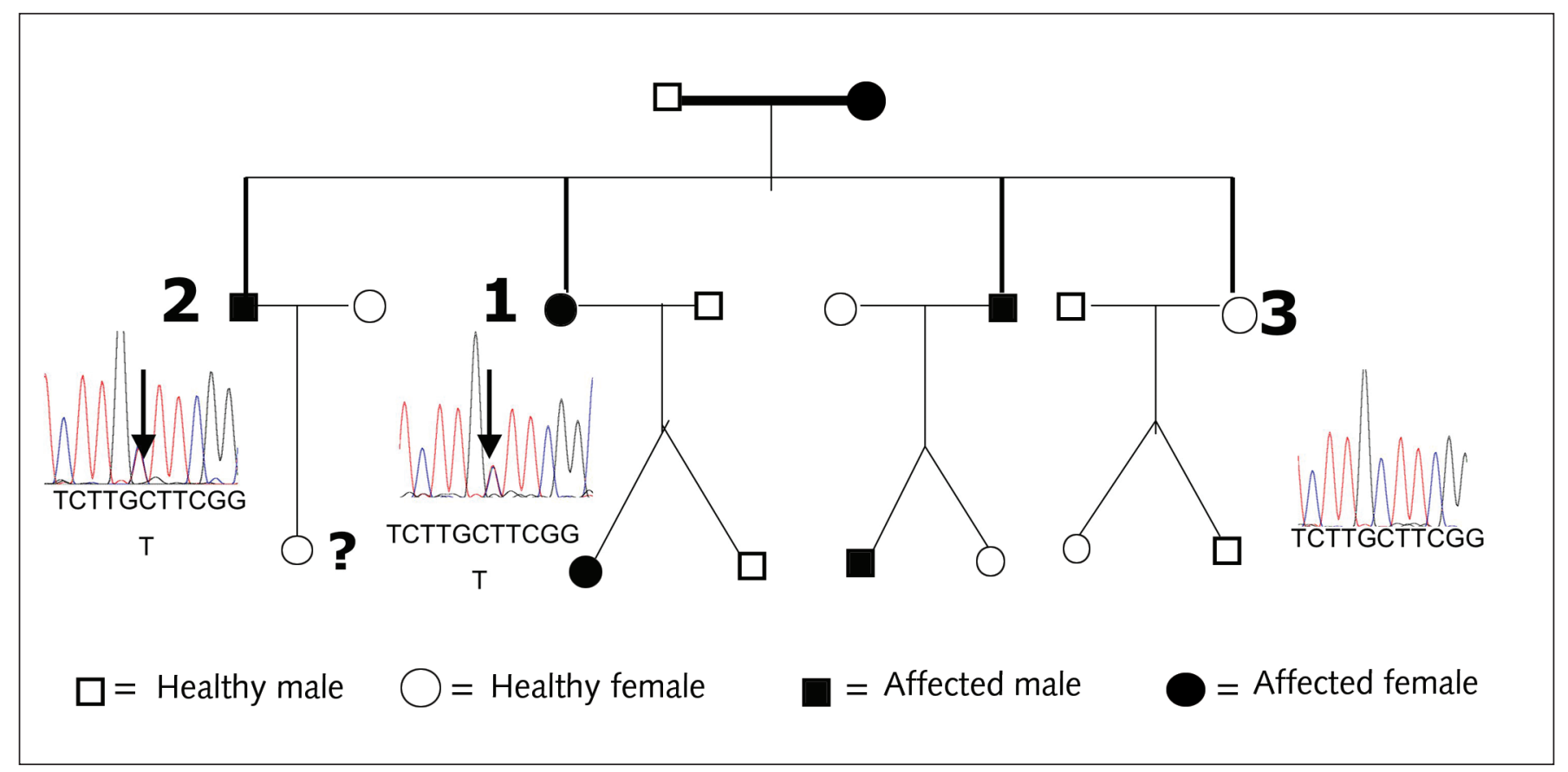

Fig. 1.- Family pedigree. Subject 1 is the index case, subject 2 is the studied affected brother, and subject 3 is the non-affected sister. The subject marked with (?) has not been studied by an ophthalmologist. Electropherograms of the L-chain ferritin sequence including the mutation are shown. Subjects 1 and 2 show a point mutation $C \rightarrow T$ (vertical arrow) which is not present in subject 3 (wild type).

Árbol genealógico de la familia afecta. El individuo 1 es el caso index y el individuo 2 su hermano afecto. El individuo 3 es otra hermana no afecta. El individuo señalado con un signo de interrogación (?) no está estudiado. Los gráficos adjuntos a cada caso corresponden al análisis molecular de la cadena $L$ del gen de la ferritina. En los individuos 1 y 2 existe una mutación por cambio $C \rightarrow T$ (flecha vertical) que no se aprecia en el individuo 3, que es wild type. 
not under the control of this regulatory system, and that is therefore independent from iron homeostasis (14). At least 11 single nucleotide polymorphisms and 4 different deletions of variable length have been identified thus far (14). The closer to the IRE loop region a mutation is located, the more severe and earlier the syndrome results. However, patients sharing the same mutation may show variability in clinical expression (7). The mutation identified in the reported family is the same as found in another family from Móstoles (Madrid) by some of us (9), but differs from that found by Pérez de Nanclares et al. (10) in a large Basque family, also in Spain $(\mathrm{A} \rightarrow \mathrm{G}$ at position 40 of IRE).

In this syndrome, hyperferritinemia results from the formation of L-chain monopolymers, or heteropolymers with a low number of $\mathrm{H}$ chains. The synthesis of $\mathrm{H}$ chains is coded for by an independent gene that is located in chromosome 5 and that is normal in this syndrome. The ability of the ferritin molecule to incorporate iron depends on its H-chain contents, and therefore ferritin molecules with excessive $\mathrm{L}$ chains in this syndrome can neither carry nor store iron. Because of this reason, no iron overload is present (4). The only known adverse consequence related to this genetic defect (i.e. cataracts) is due to the entrance and accumulation of $\mathrm{L}$ chains into the lens of affected subjects, which may reach values up to 15 (15) or even 1500 (7) above the normal range. The early formation of cataracts is difficult to explain based only on a deposition of $\mathrm{L}$ chains, because they are soluble; it has been suggested that they would affect the solubility of other proteins or deteriorate the antioxidant defenses of the lens (15).

The incidence of this syndrome is low, but as many clinicians are becoming aware of its existence, more reports on newly discovered families are being published $(7,8)$. However, an epidemic is not to be expected. In a study on 3249 blood donors, Bozzini et al. (16) identified 13 subjects with plasma ferritin values $>300 \mathrm{ng} / \mathrm{mL}$, and none of them carried mutations at the IRE region of the L-chain gene. Nevertheless, this syndrome should be known by clinicians dealing with hyperferritinemia (i.e. hepatologists and hematologists) to avoid unnecessary and even dangerous phlebotomies, as happened in the patient we report.

\section{REFERENCES}

1. Ponka P, Beaumont C, Richardson DR. Function and regulation of transferrin and ferritin. Semin Hematol 1998; 35: 35-54.

2. Feder JN, Gnirke A, Thomas W, Tsuchihashi Z, Ruddy DA, Basava A, et al. A novel MHC class-I like gene is mutated in patients with hereditary haemochromatosis. Nat Genet 1996; 13: 399-408.

3. Zúñiga A, Orera MA. Genética de las sobrecargas férricas. An Med Interna 2002; 19: 195-201.

4. García-Herce JA, Salvador C. Genética de las sobrecargas y el síndrome congénito de hiperferritinemia y cataratas. An Med Interna 2003: 20: 57-8.

5. Girelli D, Olivieri O, de Franceschi L, Corrocher R, Bergamaschi G, Cazzola M. A linkage between hereditary hyperferritinaemia not related to iron overload and autosomal dominant congenital cataract. Brit J Haematol 1995; 90: 931-4.

6. Beaumont C, Leneuve P, Devaux I, Scoazec J-Y, Berthier M, Loiseau $\mathrm{M}-\mathrm{N}$, et al. Mutation in the iron responsive element of the L-ferritin mRNA in a family with dominant hyperferritinaemia and cataract. Nature Genet 1995; 11: 444-6.

7. Girelli D, Bozzini C, Zecchina G, Tinazzi E, Bosio S, Piperno A, et al. Clinical, biochemical and molecular findings in a series of families with hereditary hyperferritinemia-cataract syndrome. Brit J Haematol 2001; 115: 334-40.

8. Hetet G, Devaux I, Soufir N, Grandchamp B, Beaumont C. Molecular analyses of patients with hyperferritinemia and normal serum iron values reveal both L ferritin IRE and 3 new ferroportin (slc11A3) mutations. Blood 2003; 102: 1904-10.

9. Cervera A, Sebastián M, Alarabe A, Díez A, Avilés MJ, Balas A Hiperferritinemia aislada en un lactante sano: síndrome hereditario de hiperferritinemia y cataratas. An Esp Pediatr 2000; 52: 267-70. (Errata 2000; 52: 568)

10. Pérez de Nanclares G, Castaño L, Martul P, Rica I, Vela A, Sanjurjo $\mathrm{P}$, et al. Molecular analysis of hereditary hyperferritinemia-cataract syndrome in a large Basque family. J Pediatr Endocrinol Metab 2001; 14: 295-300.

11. McLeod JL, Craig J, Gumley S, Roberts S, Kirkland MA. Mutation spectrum in Australian pedigrees with hereditary hyperferritinaemiacataract syndrome reveals novel and de novo mutations. Brit J Haematol 2002; 118: 1179-82.

12. Balas A, Avilés MJ, García-Sánchez F, Vicario JL, Cervera A. Description of a new mutation in the L-ferritin iron-responsive element associated with hereditary hyperferritinemia-cataract syndrome in a Spanish family. Blood 1999; 93: 4020-1.

13. Online Mendelian Inheritance in Man (OMIM). 600886: Hyperferritinemia-cataract syndrome. http://www.ncbi.nlm.nih.gov/omim/

14. Roetto A, Bosio S, Gramaglia E, Barilaro MR, Zecchina G, Camaschella C. Pathogenesis of hyperferritinemia cataract syndrome. Blood Cells Mol Dis 2002; 29: 532-5.

15. Levi S, Girelli D, Perrone F, Pasti M, Beaumont C, Corrocher R, et al. Analysis of ferritins in lymphoblastoid cell lines and in the lens in subjects with hereditary hyperferritinemia-cataract syndrome. Blood 1998; 91: 4180-7.

16. Bozzini C, Galbiati S, Tinazzi E, Aldigeri R, De Matteis G, Girelli D. Prevalence of hereditary hyperferritinemia-cataract syndrome in blood donors and patients with cataract. Hematologica 2003; 88: 219-20. 


\title{
Síndrome hereditario de hiperferritinemia y cataratas. Descripción de una nueva familia en España
}

\author{
J. M. Ladero, A. Balas ${ }^{1}$, F. García-Sánchez ${ }^{1}$ J. L. Vicario ${ }^{1}$ y M. Díaz-Rubio

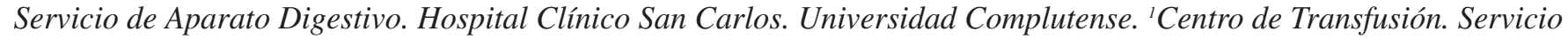 \\ Madrileño de Salud. Consejería de Sanidad. CAM. Madrid
}

\section{RESUMEN}

El síndrome de hiperferritinemia y cataratas es un trastorno autosómico dominante debido a mutaciones en la región 5' no codificante del gen de la cadena ligera de ferritina, localizado en 19q.3-q13,4. Como consecuencia se altera la morfología de la region IRE (iron responsive element) que pierde su capacidad normal de regular la síntesis de cadenas ligeras de ferritina, las cuales se sintetizan en exceso y forman complejos que se acumulan en el cristalino, dando lugar a cataratas precoces.

Se presenta una familia española con 7 miembros afectados en tres generaciones. El estudio genético pone de manifiesto un cambio de una sola base $\mathrm{C} \rightarrow \mathrm{T}$ en posición 33 del IRE en el caso index y un hermano, mientras que otra hermana no afecta del síndrome no mostraba mutaciones.

Este síndrome se identificó en 1995 y aún es poco conocido. La sospecha clínica debe surgir ante sujetos con cataratas precoces, especialmente si son de tipo familiar, o en personas con cifras elevadas de ferritina sin causa aparente y sin signos de sobrecarga de hierro. El trastorno no parece tener otras consecuencias que las cataratas y su identificación permite establecer un pronóstico favorable y evitar sangrías terapéuticas innecesarias.

Palabras clave: Hiperferritinemia. Cataratatas. Cadenas L de ferritina. Proteínas reguladoras del hierro.

\section{INTRODUCCIÓN}

La hiperferritinemia de intensidad variable es un hallazgo bastante frecuente puesto que la ferritina es un reactante de fase aguda cuya síntesis está aumentada por efecto de determinadas citocinas que se liberan en el curso de procesos inflamatorios y neoplásicos (1). La hiperferritinemia extrema es un rasgo característico, aunque no patognomónico ni obligado, de la hemocromatosis hereditaria, asociada normalmente a una elevación de la sideremia y del índice de saturación de transferrina. La identificación de las mutaciones del gen HFE, especialmente de la $\mathrm{C} 282 \mathrm{Y}$ en homocigosis o en heterocigosis mixta con la H63D, permite identificar con certeza más del 90\% de los casos de hemocromatosis genética en caucásicos (2). Otras causas genéticas de sobrecarga de hierro han sido revisadas recientemente (3), aunque con matizaciones (4).
En 1995 Girelli y cols. en Italia (5) y Bonneau y cols. en Francia (6) identificaron un síndrome que asociaba hiperferritinemia con cataratas de aparición precoz, con un patrón hereditario autosómico dominante, no asociado a sobrecarga de hierro. Desde entonces se han identificado varias familias en diferentes lugares de Europa $(7,8)$, incluida España $(9,10)$ y del resto del mundo $(11)$, si bien se considera que el trastorno es bastante raro. Presentamos una nueva familia en la que se ha identificado esta alteración.

\section{OBSERVACIÓN CLÍNICA}

El caso índice es una mujer española de raza blanca, natural y residente en Madrid. Cuando consultó por primera vez con nosotros (mayo 2003) tenía 44 años. A los 20 años había sido diagnosticada de cataratas e intervenida a los 33. A los 42 años, en un análisis de empresa, se detectó por primera vez hiperferritinemia que se confirmó en un segundo análisis $(1130 \mu \mathrm{g} / \mathrm{l})$. El resto de la analítica habitual era completamente normal, con hemoglobina $12,4 \mathrm{~g} / \mathrm{dl}$, sideremia $74 \mu \mathrm{g} / \mathrm{dl}$, IST $17 \%$, marcadores virales $\mathrm{B}$ y $\mathrm{C}$, autoanticuerpos no organoespecíficos, cobre, ceruloplasmina y $\alpha-1$ antitripsina normales o negativos. Ecografía abdominal normal. La enferma era heterocigota simple para la mutación H63D del gen HFE. Remitida a su especialista, se le prescribieron cinco sangrías de $300 \mathrm{ml}$ en un periodo de 4 meses, al cabo de los cuales la ferritina era de $1.050 \mu \mathrm{g} / \mathrm{l}$, con sideremia $46 \mu \mathrm{g} / \mathrm{dl}$, IST $12 \%$ y hemoglobina $10,9 \mathrm{~g} / \mathrm{dl}$. La enferma fue remitida a la consulta externa de nuestro servicio.

En la anamnesis refirió que su madre y dos de sus tres hermanos habían sido diagnosticados de cataratas en la juventud y posteriormente de hiperferritinemia. La enferma tenía dos hijos, uno de los cuales tenía cataratas e hiperferritinemia. Lo mismo ocurría con uno de los dos hijos de uno de sus dos hermanos afectados. El otro hermano afectado tenía una hija de 8 años que no había sido estudiada. La hermana no afectada tenía dos hijos normales (Fig. 1). 
Se confirmó la hiperferritinemia y la absoluta normalidad del resto de los parámetros analíticos férricos, hepáticos y hemáticos. Se realizó estudio genético de la paciente index y de dos de sus hermanos, uno afectado y el otro no. El método utilizado ha sido el mismo que se utilizó en otra familia portadora del síndrome e identificada con anterioridad (9), según se ha descrito en otro lugar (12). Tanto el caso index como el hermano afectado eran portadores de un cambio de una sola base $\mathrm{C} \rightarrow \mathrm{T}$ en la posición 33 del IRE (iron responsive element) del gen de la cadena ligera de ferritina. La hermana no afectada no presentaba mutaciones.

\section{COMENTARIO}

El síndrome hereditario de cataratas e hiperferritinemia es un trastorno autosómico dominante debido a mutaciones en la región 5', no codificante, del gen de la cadena corta de ferritina, que se localiza en 19q13.3-q13.4 (13). Esta región se denomina IRE (iron responsive element: elemento que responde al hierro) y su función es unirse a determinadas proteínas citoplásmicas denominadas IRP (iron regulatory proteins: proteínas reguladoras del hierro). En caso de deficiencia de hierro las IRP adquieren una afinidad elevada por el IRE e inhiben la síntesis de cadenas ligeras de ferritina. La interacción IREIRP se produce sobre una estructura anular formada por cinco pares de bases en un extremo del IRE (14).

Las mutaciones que alteran o influyen sobre la morfología de esta región anular impiden o disminuyen el grado de interacción IRE-IRP, lo que permite una síntesis de cadenas ligeras de ferritina no sujeta al control regulador generado por este sistema y que por lo tanto es independiente del estado homeostático del hierro (14). Se han identificado hasta el momento al menos 11 mutaciones por cambio de una sola base (SNPs) y 4 deleciones de extensión variable (14). Cuanto más próxima esté la mutación de la región anular del IRE mayor y más precoz será el trastorno, aunque existe amplia variabilidad entre suje- tos portadores de la misma mutación (7). La mutación identificada en la familia que presentamos es la misma que se identificó en una familia de Móstoles (Madrid) por este mismo grupo (9), pero difiere de la registrada por Pérez de Nanclares y cols. (10) en una extensa familia vasca $(A \rightarrow G$ en posición 40$)$ de la zona IRE.

La hiperferritinemia propia de este síndrome se debe a la formación de homopolímeros de cadenas L, o de heteropolímeros con pequeñas cantidades de cadenas $\mathrm{H}$ de ferritina, cuya síntesis depende de otro gen situado en el cromosoma 5 y que no está afectado en este síndrome. La capacidad de incorporación de hierro a la ferritina depende de su riqueza en cadenas $\mathrm{H}$, por lo que las moléculas de ferritina generadas en este síndrome, como tienen un gran exceso de cadenas L, apenas transportan ni acumulan hierro, lo que impide que originen sobrecarga de este metal. Únicamente la entrada y acumulación de cadenas L en el cristalino de los sujetos afectados, que puede llegar a ser 15 veces mayor que en individuos normales según algunos autores (15) o hasta 1.500 según otros (7), origina cataratas precoces, que no se explican solamente por el exceso de cadenas L, que son solubles, por lo que se ha sugerido que estas cadenas podrían afectar a la solubilidad de otras proteínas o deteriorar los mecanismos antioxidantes (15).

La frecuencia de este síndrome es baja, pero conforme se va conociendo su existencia por parte de los clínicos aumentan las comunicaciones de familias afectadas (7, 8). No obstante no es de esperar una "epidemia". Bozzini y cols. (16) identificaron 13 donantes de sangre con ferritinemia $>300 \mu \mathrm{g} / \mathrm{l}$ de un total de 3.249, 15 individuos con catarata precoz (antes de los 40 años) y 26 sujetos con catarata y ferritina $>300 \mu \mathrm{g} / \mathrm{l}$, y en ningún caso detectaron mutaciones de la región IRE del gen de la cadena L de ferritina. De todos modos la existencia de este síndrome debe ser conocida por los clínicos que con mayor frecuencia detectan enfermos con hiperferritinemia (hematólogos y hepatólogos) con el fin de evitar la práctica de sangrías innecesarias e inconvenientes, como ocurrió en el caso index que presentamos. 